\title{
The Value of Accurate Forecasts and a Probabilistic Method for Robust Scheduling of Residential Distributed Energy Resources
}

\author{
Michael Angelo A. Pedrasa ${ }^{1,2}$, Ted D. Spooner ${ }^{1}$ and Iain F. MacGill ${ }^{1}$ \\ ${ }^{1}$ School of Electrical Engineering and Telecommunications, and Centre for Energy and Environmental Markets, \\ University of New South Wales, Sydney, Australia \\ ${ }^{2}$ Electrical and Electronics Engineering Institute, University of the Philippines, Quezon City, Philippines
}

\begin{abstract}
We describe a decision-support tool that optimizes the energy services of residential end-users by scheduling the operation of available distributed energy resources. We discuss the application of the tool to a 'smart' home case study and the solution to the resulting highly-dimensional scheduling problem. We then use the optimal schedules formulated by the tool to determine the value of the forecasted information used when the schedules are created. This is achieved by computing the additional costs avoided by the end-users due to the accuracy of the forecasts. We also demonstrate how to use the tool to derive robust schedules when the end-users are not certain on the magnitude of solar insolation, magnitude of energy service demands, availability of a plug-in hybrid vehicle as storage, and status of Critical Peak Pricing. The robust schedule is derived by maximizing the expected net benefit when the schedule is applied to all likely scenario outcomes.
\end{abstract}

\section{INTRODUCTION}

A 'smart' home is a residential building equipped with smart appliances and sensors that are designed to achieve goals that benefit end-users by coordinating with each other. One such goal is to manage energy consumption while providing the energy services the end-users require. Examples of residential energy services are space conditioning, illumination, food storage and preparation, and hot water, among others.

Several hardware and software platforms that enable a 'smart' home have been proposed. Examples are a remotecontrollable power outlet system [1], a microcontroller-based electricity consumption warning system [2], and a system that enables local and remote control (using the internet or telephone) of appliances [3]. In these systems, much of the decision-making with respect to the operation of the appliances are done by the end-users.

We proposed in an earlier paper [4] a decision-support tool that may be used in a 'smart' home. The decision-support tool optimizes the end-user energy services by scheduling the operation of residential distributed energy resources (DER) like appliances and storage devices. The decision-support tool is based upon the end-users assigning benefit to the energy services, and the maximization of the net benefits that the endusers derive from the services. We used a 'smart' home case study to demonstrate the decision-support tool.

Several papers have also presented methods on how to schedule residential DER [5]-[7]. The main difference of our proposed tool is we recognize that end-users put different levels of benefit to different services at different times of the day, and we used these benefits to formulate the DER schedules. This approach also enables the curtailment of services if the cost of provision exceeds their benefits.

The end-users utilize forecasted information when they create the DER schedules. In this paper, we determine the value of making accurate forecasts. We also present an approach to making robust schedules when in possession of inaccurate forecasts. We demonstrate our approaches using an extended version of the case study in [4].

The decision-support tool, the 'smart' home case study, and the related optimization problem and solution are described in Section II, the value of accurate forecasts is described in Section III, the approach to formulating robust schedules is discussed in Section IV, and our conclusions are summarized in Section V.

\section{Optimal DER Scheduling in A 'SMART' Home}

\section{A. Decision-Support Tool}

The decision-support tool [4] is composed of an energy service model and a DER scheduling algorithm (or scheduler). The energy service model enables end-users to assign monetary benefit to the energy services, and assigns this benefit to the energy that realizes the energy service (or the "energy equivalent" of the service). The model also represents the temporal variation of the demand for and value of the services.

The DER scheduler maximizes the net benefit derived from the services by scheduling the operation of DER. The net benefit is equal to the total benefits derived from the services less the cost of energy consumption. The scheduler determines the DER schedule $\boldsymbol{x}$ that maximizes

$$
=\sum_{t=1}^{T} \sum_{i=1}^{S}\left[\lambda_{\mathrm{ES}, i}(t) \cdot U_{\mathrm{ES}, i}(t, \boldsymbol{x})\right]-\text { Cost }
$$

where $\lambda_{\mathrm{ES}, i}$ is the monetary benefit assigned to each unit of "energy equivalent" of the $i^{\text {th }}$ service, and $U_{\mathrm{ES}, i}$ is the demand for the "energy equivalent" of the $i^{t h}$ service. The summation term adds up the benefits derived from all services and subtracted from it is the cost (Cost) of electricity consumption. We used co-evolutionary particle swarm optimization (PSO) 


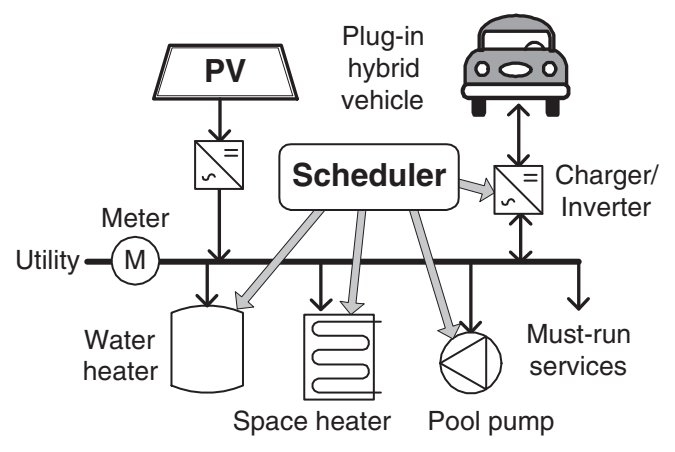

Fig. 1. The 'smart' home case study.

with stochastic repulsion among particles [8] to solve (1). We chose PSO because it can generate feasible solutions within managable computation times and because of its ease in implementation.

\section{B. The 'Smart' Home Case Study}

The case study involves the scheduling of four DER to optimize the provision of energy services in a 'smart' home, as shown in Fig. 1. Over an entire 24-hour period, the scheduler determines (a) the charging rate of the plug-in hybrid vehicle (PHEV) $x_{\mathrm{EV}}$, (b) the heating power of the space heater $\boldsymbol{x}_{\mathrm{SH}}$, (c) the hours when to run the pool-pump $\boldsymbol{x}_{\mathrm{PP}}$, and (d) the hours when to turn on the water heater $\boldsymbol{x}_{\mathrm{WH}}$. These DER provide the PHEV charging, space heating, pool pumping, and hot water services. All other services are lumped together into a mustrun service and this includes illumination, food storage and preparation, and entertainment, among others.

Based on (1), the DER scheduler would determine the DER schedule $\boldsymbol{x}=\left[\begin{array}{llll}\boldsymbol{x}_{\mathrm{EV}} & \boldsymbol{x}_{\mathrm{SH}} & \boldsymbol{x}_{\mathrm{WH}} & \boldsymbol{x}_{\mathrm{PP}}\end{array}\right]$ that maximizes

$$
\sum_{t=1}^{T}\left(\begin{array}{c}
\lambda_{\mathrm{MR}}(t) U_{\mathrm{MR}}(t)+\lambda_{\mathrm{EV}}(t) U_{\mathrm{EV}}\left(t, \boldsymbol{x}_{\mathrm{EV}}\right) \\
+\lambda_{\mathrm{SH}}(t) U_{\mathrm{SH}}\left(t, \boldsymbol{x}_{\mathrm{SH}}\right)+\lambda_{\mathrm{WH}}(t) U_{\mathrm{WH}}\left(t, \boldsymbol{x}_{\mathrm{WH}}\right) \\
+\lambda_{\mathrm{PP}}(t) U_{\mathrm{PP}}\left(t, \boldsymbol{x}_{\mathrm{PP}}\right)-r_{\mathrm{elec}}(t) P_{\mathrm{e}}(t, \boldsymbol{x})
\end{array}\right)
$$

where $\lambda_{\text {service }}$ is the monetary benefit assigned to each unit of "energy equivalent", $U_{\text {service }}$ is the hourly demand for the "energy equivalent" of the services, $r_{\text {elec }}$ is the cost of each $\mathrm{kWh}$ of electricity consumption, and $P_{\mathrm{e}}$ is the total electricity consumption every hour.

$$
P_{\text {elec }}=P_{\mathrm{MR}}+P_{\mathrm{EV}}+P_{\mathrm{SH}}+P_{\mathrm{WH}}+P_{\mathrm{PP}}-P_{\mathrm{PV}}
$$

The demand for the energy services and the monetary benefit assigned to their "energy equivalents" are shown in Fig. 2. For the space heating service, the actual temperature should be within $1 \mathrm{C}^{\circ}$ from the desired temperature. The PHEV battery should be fully charged at $8 \mathrm{AM}$, and the pool pump should run at most 6 hours.

The energy service model relates the electricity consumption of the energy service, $P_{\text {service }}$, to the "energy equivalent," $U_{\text {service}}$, of the service. The "energy equivalent" of the space and water heating services are the thermal energy content of indoor air and hot water, while the "energy equivalent" of the PHEV charging service is the energy stored in the battery. (a)
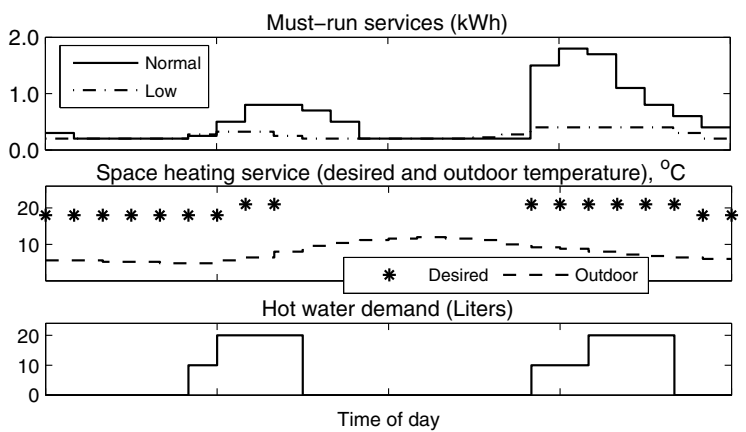

Time of day

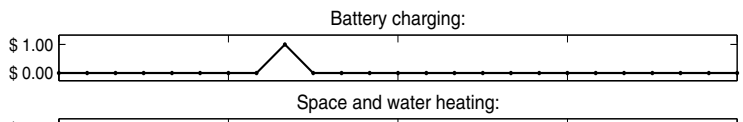

(b)

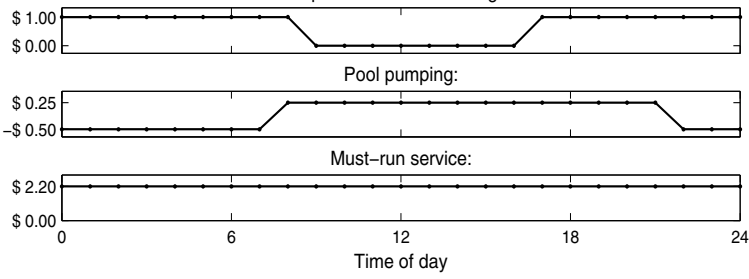

Fig. 2. Energy service models: (a) hourly demand for the energy services, (b) benefit assigned to each kWh of "energy equivalent" $\left(\lambda_{\mathrm{ES}}(t)\right)$.

Physically-based models were used to relate the "energy equivalent" of these services to the hourly energy consumption. For the must-run and pool pumping services, the actual energy consumption is taken as the "energy equivalent."

\section{DER Scheduling Using Particle Swarm Optimization}

We solved the DER schedules using co-evolutionary particle swarm optimization (PSO) with stochastic repulsion among the particles. PSO is a heuristic population-based optimization algorithm that searches for the solution by allowing candidate solutions (or particles) to wander around the solution space while the particles communicate with each other [9]. The particles tend to move towards the best performing particle (global best) and the best position it has ever achieved (personal best).

Equation (2) is a highly-dimensional optimization problem, hence we used a co-evolutionary version of PSO [10]. Coevolutionary PSO employs a divide-and-conquer approach: it breaks the vector to be optimized into several component vectors and a swarm optimizes each component. We further improved the performance of co-evolutionary PSO by incorporating stochastic repulsion among the particles [8]. In our approach, the particles move away from the personal and global best positions on some iterations, conditional to a probability that is a function of the iteration number.

Some of the decision variables are binary, like the turning on and off of the water heater and the pool pump, so we used the binary version of PSO [11] to optimize these schedules.

\section{VAlue of AcCurate Forecasts}

The end-users use predictions such as their planned behavior and weather forecasts when they use the scheduler to formulate the DER schedules. In the case study, the DER 
schedules are created based on the number of people that might be present during the day, their plans on using the PHEV when they leave, the solar insolation forecast made available by the weather bureau, and their prediction of the status of Critical Peak Pricing (CPP). These information are used because the occupancy levels would affect the demand for the energy services, the PHEV may be used as a storage DER if the end-users leave it at home, the solar insolation affects the amount of PV generation, and the total electricity demand should be reduced during an active CPP period. Therefore, the scheduling of DER will only be effective if the forecasts used to generate the schedules are accurate.

It is sometimes inevitable that the end-users make incorrect predictions. They could plan to use the PHEV but decide on the day to leave it instead. In this case, they might lose some benefit because the PHEV could have been used as storage DER. Another potentially costly outcome is that the utility activates CPP for the day, and the DER are scheduled with CPP not assumed.

In this section, we determine the value of making accurate forecasts. This is achieved by implementing schedules optimized for other scenarios to the scenario that turned out, and determining the additional costs incurred by the end-users due to the incorrect implemented schedules. The magnitude of the avoided costs if the correct schedule is implemented are indicators of the value of the accurate forecasts.

In this paper, the cost incurred by the user is equal to the cost of electricity consumption added to the costs of any undelivered services, less the value of the energy stored in the storage DER at the end of the day. We used this formula because it takes into account the benefits not gained by the end-user when services are not provided, and the stored energy which reduces the cost for the succeeding day.

In the case study, we assumed two possible states each for the expected solar insolation, expected demand for must-run and hot water services, availability of the PHEV as storage DER, and status of CPP. A scenario is a combination of the outcomes of the 4 different states so there are 16 possible scenarios. For brevity, we use an 8-character code to refer to a scenario: $\mathrm{S} x \mathrm{D} x \mathrm{~B} x \mathrm{C} x$, where $x$ is either 0 or 1 . The letters $\mathrm{S}$, $\mathrm{D}, \mathrm{B}$ and $\mathrm{C}$ refer to the solar insolation, magnitude of service demand, availability of the PHEV, and CPP status. Table I summarizes the different meanings of $x$ for each condition. To illustrate, scenario S1D1B1C0 pertains to a sunny day, demand for must-run and hot water services are normal, the PHEV is parked from $8 \mathrm{AM}$ to $5 \mathrm{PM}$, and $\mathrm{CPP}$ is not active.

We used the energy rates shown in Table II and investigated the cases when feed-in energy is compensated at ToU rates and not compensated at all.

The results are shown in Table III for tariff ToU energy + ToU feed-in. A row corresponds to the scenario that turned out and a column corresponds to the scenario to which the implemented schedule is optimized. The diagonal entries, therefore, are the actual costs (\$) when the correct schedules are implemented, and these are the lowest costs that may be achieved for the corresponding scenarios. The off-diagonal
TABLE I

DESCRIPTION OF SCENARIO CODE SXDXBXCX

\begin{tabular}{l|c|c}
\hline \multicolumn{1}{c|}{ Condition } & \multicolumn{2}{|c}{ Value of $x$} \\
\cline { 2 - 3 } & 1 & 0 \\
\hline S: Solar insolation & Sunny & Cloudy \\
D: Demand for must-run and hot water service & Normal & Low ${ }^{1}$ \\
B: PHEV available as storage DER (8 AM to 5 PM) & Yes & No \\
C: Critical Peak Pricing & Yes & No \\
\hline
\end{tabular}

${ }^{1}$ Refer to Fig. 2(a) for the must-run and hot water services. Low demand for hot water service is $25 \%$ of the demand shown in the figure.

TABLE II

ELECTRICITY TARIFF

\begin{tabular}{l|c}
\hline \multicolumn{1}{c|}{ Time-of-Use (ToU) Interval } & Rate $(\$ / \mathrm{kWh})$ \\
\hline Peak: $2-8 \mathrm{PM}$ & 0.3564 \\
Shoulder: $7 \mathrm{AM}-2 \mathrm{PM}, 8-10 \mathrm{PM}$ & 0.1408 \\
Off-peak: 10 PM - 7 AM & 0.0814 \\
\hline Critical peak price (CPP): 5 - 8 PM & 2.000 \\
\hline
\end{tabular}

entries are the additional costs incurred by the end-users due to the incorrect forecasts, expressed as percent increase with respect to the best costs (or diagonal entries). The entries with superscripts are those which the implemented schedule differs by one state to the scenario that turn out, that is, the end-users made one inaccurate prediction of the four uncertain events. All other off-diagonal entries are the costs when the end-users made two or more incorrect predictions.

The table contains a large amount of information so we use a simple case to demonstrate how the data are interpreted. In Table III, let us assume that the day turned out to be sunny, service demands are normal, PHEV is available as storage, and CPP is not active (S1D1B1C0, row 1). The cost is $\$ 3.91$ if the end-users made accurate forecasts, and therefore implemented the correct schedule. If the solar insolation forecast is incorrect, they implement the schedule for S0D1B1C0 (column 2) and the cost incurred is also $\$ 3.91$. There is no additional cost ( $0 \%$ in the table) so there is no value in making accurate solar insolation forecasts. If the end-users made an incorrect demand forecast, they implement the schedule for S1D0B1C0 (col. 3), and the cost would increase by $90 \%$. Using similar reasoning, making an incorrect PHEV availability forecast would result in the cost to increase by $15 \%$ and an incorrect CPP status forecast would also result in the cost to increase by $15 \%$.

Using the results, we conclude for tariff ToU energy + ToU feed-in that:

1) There is no value in making accurate solar insolation forecasts. It brings no benefit because the value of each $\mathrm{kWh}$ of PV generation is the same whether it is consumed within the house or exported to the grid.

2) The value of correct demand forecast is significant especially when the expected demand is normal. It is significant because if a schedule for low demand is implemented, the water heater is not turned on long enough to heat the required hot water for the day. The additional cost is mostly due to the required hot water that is not available.

3) The value of knowing the availability of the PHEV is 
TABLE III

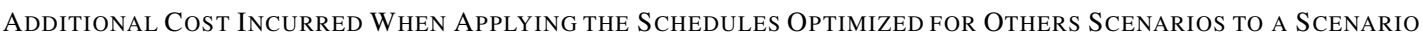

Tariff: ToU energy + ToU feed-in. The diagonal entries are actual costs, in \$, while the off-diagonal entries are the additional costs due to the implementation of the incorrect schedule. The additional costs are expressed as \% increase with respect to the diagonal entries on the same row, rounded to the nearest $5 \%$.

\begin{tabular}{|c|c|c|c|c|c|c|c|c|c|c|c|c|c|c|c|c|}
\hline \multirow{3}{*}{$\begin{array}{c}\text { Actual } \\
\text { Scenario }\end{array}$} & \multicolumn{16}{|c|}{ Scenario corresponding to the implemented schedule } \\
\hline & 1 & 2 & 3 & 4 & 5 & 6 & 7 & 8 & 99 & 10 & 11 & 12 & 13 & 14 & 15 & 16 \\
\hline & $\mathrm{B} 1 \mathrm{C} 0$ & $\mathrm{~B} 1 \mathrm{C} 0$ & $\mathrm{~B} 1 \mathrm{C} 0$ & $\mathrm{~B} 1 \mathrm{C} 0$ & B0C0 & B0C0 & B0C0 & B0C0 & B1C1 & B1C1 & B1C1 & $\mathrm{B} 1 \mathrm{C} 1$ & B0C1 & B0C1 & B0C1 & B0C1 \\
\hline $2 \mathrm{~S} 0 \mathrm{D} 1 \mathrm{~B} 1 \mathrm{C} 0$ & $0 \%{ }^{\mathrm{S}}$ & $\$ 6.01$ & $60 \%$ & $60 \%{ }^{\mathrm{D}}$ & $10 \%$ & $10 \%^{\mathrm{B}}$ & $70 \%$ & $70 \%$ & $10 \%$ & $10 \%{ }^{\mathrm{C}}$ & $60 \%$ & $60 \%$ & $20 \%$ & $20 \%$ & $80 \%$ & $80 \%$ \\
\hline $3 \mathrm{~S} 1 \mathrm{D} 0 \mathrm{~B} 1 \mathrm{C} 0$ & $0 \%^{\mathrm{D}}$ & $5 \%$ & $\$ 1.44$ & $0 \% \mathrm{~S}$ & $40 \%$ & $40 \%$ & $35 \%{ }^{\mathrm{B}}$ & $35 \%$ & $45 \%$ & $45 \%$ & $0 \%{ }^{\mathrm{C}}$ & $0 \%$ & $80 \%$ & $80 \%$ & $80 \%$ & $75 \%$ \\
\hline $4 \mathrm{SOD} 0 \mathrm{~B} 1 \mathrm{C} 0$ & $0 \%$. & $0 \%$ D & $0 \%{ }^{\mathrm{s}}$ & $\$ 3.54$ & $15 \%$ & $15 \%$ & $15 \%$ & $15 \%^{\mathrm{B}}$. & $15 \%$ & $15 \%$ & $0 \%$ & $0 \%$ C & $30 \%$ & $30 \%$ & $30 \%$ & $30 \%$ \\
\hline 7 S1D0B0C0 & $0 \%$ & $0 \%$ & $0 \%^{\mathrm{B}}$ & $0 \%$ & $0 \%^{\mathrm{D}}$ & $0 \%$ & $\$ 2.31$ & $0 \% \mathrm{~s}$ & $25 \%$ & $25 \%$ & $0 \%$ & $0 \%$ & $25 \%$ & $25 \%$ & $25 \% \mathrm{C}$ & $25 \%$ \\
\hline 8 SOD0B0C0 & $0 \%$ & $0 \%$ & $0 \%$ & $0 \%^{\mathrm{B}}$ & $0 \%$ & $0 \%{ }^{\mathrm{D}}$ & $0 \% \mathrm{~s}$ & $\$ 4.41$ & $15 \%$ & $15 \%$ & $0 \%$ & $0 \%$ & $15 \%$ & $15 \%$ & $15 \%$ & $15 \%^{\mathrm{C}}$ \\
\hline 9 S1D1B1C1 & $100 \% \mathrm{C}^{\mathrm{C}}$ & $95 \%$ & $150 \%$ & $155 \%$ & $110 \%$ & $105 \%$ & $155 \%$ & $155 \%$ & $\$ 7.06$ & $0 \% \mathrm{~s}^{\mathrm{s}}$ & $95 \% \mathrm{D}^{\mathrm{D}}$ & $95 \%$ & $95 \%$ & $95 \%$ & $145 \%$ & $145 \%$ \\
\hline 10 S0D1B1C1 & $75 \%$ & $75 \%^{\mathrm{C}}$ & $115 \%$ & $120 \%$ & $85 \%$ & $80 \%$ & $120 \%$ & $120 \%$ & $0 \%{ }^{\mathrm{s}}$ & $\$ 9.15$ & $75 \%$ & $75 \%^{\mathrm{D}}$ & $75 \%$ & $70 \%{ }^{\mathrm{B}}$ & $110 \%$ & $110 \%$ \\
\hline 11 S1D0B1C1 & $260 \%$ & $255 \%$ & $270 \%^{\mathrm{C}}$ & $285 \%$ & $315 \%$ & $300 \%$ & $290 \%$ & $290 \%$ & $70 \%^{\mathrm{D}}$ & $70 \%$ & $\$ 1.47$ & $0 \%{ }^{\mathrm{S}}$ & $240 \%$ & $240 \%$ & $240 \%^{\mathrm{B}}$ & $240 \%$ \\
\hline 12 S0D0B1C1 & $110 \%$ & $105 \%$ & $110 \%$ & $120 \%^{\mathrm{C}}$ & $130 \%$ & $125 \%$ & $120 \%$ & $120 \%$ & $30 \%$ & $30 \%^{\mathrm{D}}$ & $0 \% \mathrm{~s}$ & $\$ 3.54$ & $100 \%$ & $100 \%$ & $100 \%$ & $100 \%^{\mathrm{B}}$ \\
\hline 16 S0D0B0C1 & $10 \%$ & $10 \%$ & $15 \%$ & $15 \%$ & $15 \%$ & $10 \%$ & $10 \%$ & $10 \%^{\mathrm{C}}$ & $0 \%$ & $0 \%$ & $10 \%$ & $15 \%^{\mathrm{B}}$ & $0 \%$ & $0 \%^{\mathrm{D}}$ & $0 \% \mathrm{~s}$ & $\$ 7.39$ \\
\hline
\end{tabular}

Entries with superscripts are those which the implemented schedule differs by one state to the scenario that turn out. A superscript $\mathrm{S}$ indicates difference in solar insolation, D indicates difference in service demand, B indicates difference in PHEV availability, and C indicates difference in CPP status.

significant especially when it is expected to be available as a storage DER. The value is significant because it can be used to store energy during the off-peak period and provide energy to other services during the peak or CPP periods.

4) The value of correct CPP forecast is significant especially when it is predicted to be active. In scenario S1D1B1C1 (row 9) for example, the grid import is minimized during the CPP period and the space heat service is not provided for an hour, as shown in Fig. 3(a). Furthermore, the PHEV discharges during this period to provide the energy required by the must-run service. These actions significantly reduce the cost incurred by the end-user. If the schedule for scenario S1D1B1C0 (column 1) is implemented (i.e. the CPP status is wrongly predicted), the additional cost is almost equal to the cost if the correct schedule is used (100\% increase). The additional cost is large because the DER were not operated to minimize the grid import during the CPP period, as shown in Fig. 3(b). The PHEV is discharged in the afternoon to achieve some energy export but the credit is not enough to lower the total cost.

We followed the same analysis in the case where feed-in energy is not compensated (due to space constraints, we are not able to present the results similar to that of Table III). We were able to conclude that:

1) The value of correct solar insolation forecast is significant. Since feed-in energy is no longer compensated, the DER should coordinate their operation so that all PV energy is consumed within the house.

2) The value of correct demand forecast is significant especially when the expected demand is normal.

3) The value of knowing the availability of the PHEV is significant especially when it is expected to be available as a storage DER.

4) The value of making a correct CPP forecast is not too significant even on cases when CPP is active. Since energy export is not compensated, the PV energy is used to pre-heat the house and the PHEV (if available) discharges to provide energy for the must-run and space heat services. These actions result in a lower grid import during the CPP period when compared to the case when feed-in is compensated, hence, the energy costs are lower.

\section{Formulating Robust DER Schedules Under FORECAST UNCERTAINTY}

The results in the previous section reveal that the end-users could incur significant additional costs if their forecasts are incorrect. In this section, we present a probabilistic method for generating robust schedules when accurate forecasts cannot be made.

The robust DER schedule is formulated by maximizing the expected net benefit. The expected net benefit is the sum of weighted evaluations of the fitness function (1) for a schedule over all likely scenarios. The end-user assumes a probability (or a confidence value) to the occurence of each scenario that may turn out, and use these probabilities as weights to the fitnesses of a schedule $\boldsymbol{x}$ applied to all scenarios. That is, if there are $\mathrm{N}$ possible scenarios, and $\pi_{n}$ is the probability (or confidence) that the $n^{\text {th }}$ scenario, $\mathrm{Sc}_{n}$, will occur, then the 
(a)

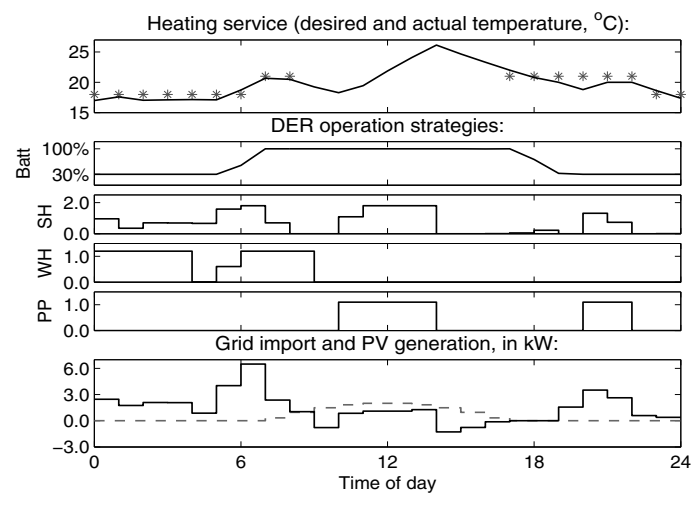

(b)

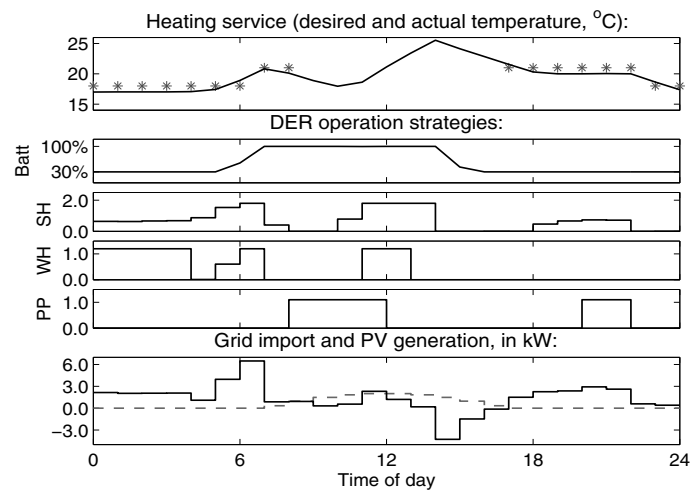

Fig. 3. DER schedules for tariff ToU energy + ToU feed-in when (a) CPP is assumed to be active and (b) CPP is assumed to be not active. Batt = PHEV battery state of charge (\% of capacity), $\mathrm{SH}=$ space heater power $(\mathrm{kW}), \mathrm{WH}$ $=$ water heater power $(\mathrm{kW}), \mathrm{PP}=$ pool pump power $(\mathrm{kW})$.

robust schedule $\boldsymbol{x}$ is determined by maximizing

$$
\sum_{n=1}^{\mathrm{N}} \pi_{n} \cdot F\left(\boldsymbol{x}, \mathrm{Sc}_{n}\right)
$$

where $F\left(\boldsymbol{x}, \mathrm{Sc}_{n}\right)$ is the fitness (or net benefit) of schedule $\boldsymbol{x}$ when applied to scenario $\mathrm{Sc}_{n}$ (evaluated using (1)), and

$$
\sum_{n=1}^{\mathrm{N}} \pi_{n}=1 .
$$

We illustrate the formulation of robust schedules using two examples. In the first example, the tariff is ToU energy + ToU feed-in and the end-users are certain that it will be sunny, the service demands will be normal and they will leave the PHEV at home. However, they are not certain about the status of CPP so they assume the probability of its occurence, $\pi_{\mathrm{C}}$. The robust schedule $x_{\mathrm{C}}$ is determined by maximizing the expected net benefit:

$$
\pi_{\mathrm{C}} \cdot F\left(\boldsymbol{x}_{\mathrm{C}}, \mathrm{S} 1 \mathrm{D} 1 \mathrm{~B} 1 \mathrm{C} 1\right)+\left(1-\pi_{\mathrm{C}}\right) \cdot F\left(\boldsymbol{x}_{\mathrm{C}}, \mathrm{S} 1 \mathrm{D} 1 \mathrm{~B} 1 \mathrm{C} 0\right) .
$$

We derive schedules for three different CPP probabilities. In the first case, the end-users are fairly sure that CPP will not be active, so they assumed a probability of $10 \%$. In the second case, they are completely unsure about the status so they assume a $50 \%$ probability. In the last case, thay are fairly sure that it will be active, so they assume a $90 \%$ probability. The robust schedules for the three CPP probabilities are shown
TABLE IV

COST INCURRED WHEN SCHEDULING UNDER CPP FORECAST UNCERTAINTY $(\$)$

\begin{tabular}{c|c|c||c|c|c}
\hline Actual & \multicolumn{5}{|c}{ Implemented schedule, assuming CPP probability $=\pi_{\mathrm{C}}$} \\
\cline { 2 - 6 } scenario $^{1}:$ & $0 \%$ & $100 \%$ & $10 \%$ & $50 \%$ & $90 \%$ \\
\cline { 2 - 6 } No CPP & $\mathbf{3 . 9 1}$ & 4.49 & 3.93 & 3.94 & 4.51 \\
With CPP & 13.99 & $\mathbf{7 . 0 6}$ & 7.67 & 7.64 & 7.06 \\
\hline Expected cost & 3.91 & 7.06 & 4.30 & 5.79 & 6.81 \\
\hline
\end{tabular}

1 The end-users are certain that it is sunny, demand is normal, and the $\mathrm{PHEV}$ is available as storage.

in Fig. 4. The robust schedules are also applied to the two possible outcomes, S1D1B1C0 (No CPP) and S1D1B1C1 (With CPP), and the costs incurred by the end-users are determined. These costs are compared to the costs resulting from schedules that assume $0 \%$ and $100 \%$ CPP probabilities. The costs are listed in Table IV.

In the robust schedules, the PHEV battery is discharged during the CPP period in all CPP probabilities. This operation reduces the grid import during the $\mathrm{CPP}$ period whether CPP is active or not. The value of the energy stored in the PHEV battery is the same whether it is exported to the grid or used to provide for other services so there is no additional cost or benefit whether the PHEV discharges to export (as shown in Fig. 3(b)) or to provide energy for other services. Furthermore, when the CPP probabilities are $10 \%$ and $50 \%$, the space heating service is provided in all hours, but when the CPP probability is $90 \%$, the total grid import during 7-8 PM is reduced and the space heating service is not provided (i.e. the actual temperature is more than $1 \mathrm{C}^{\circ}$ from the required temperature).

The entries in the first two columns of Table IV are derived from Table III. The expected cost is closer to $\$ 3.91$ when the CPP probability is $10 \%$, but closer to $\$ 7.06$ when the CPP probability is $90 \%$. The schedule when the CPP probability is $50 \%$ resulted in actual costs (\$3.94 and \$7.64) that are quite close to the best cost values $(\$ 3.91$ and $\$ 7.06)$. The robust schedules were able to hedge the end-users from large additional costs that could result from inaccurate predictions.

In the second example, the tariff is ToU energy + no feedin and the end-users are planning to make the car available as storage DER, and CPP is not active. They are not certain about the solar insolation and the service demands, however, they are leaning towards predicting a sunny day and having normal service demands. They assume a probability of $80 \%$ for sunny day and $60 \%$ for normal service demands. There are four likely scenarios and their probabilities are determined by multiplying the insolation probability to the demand magnitude probability. The scenario probabilities are listed in Table V.

The robust schedule $\boldsymbol{x}_{\mathrm{R}}$ is determined by maximizing the expected net benefit:

$$
\begin{aligned}
& 0.48 F\left(\boldsymbol{x}_{\mathrm{R}}, \mathrm{S} 1 \mathrm{D} 1 \mathrm{~B} 1 \mathrm{C} 0\right)+0.32 F\left(\boldsymbol{x}_{\mathrm{R}}, \mathrm{S} 1 \mathrm{D} 0 \mathrm{~B} 1 \mathrm{C} 0\right) \\
& +0.12 F\left(\boldsymbol{x}_{\mathrm{R}}, \mathrm{S} 0 \mathrm{D} 1 \mathrm{~B} 1 \mathrm{C} 0\right)+0.08 F\left(\boldsymbol{x}_{\mathrm{R}}, \mathrm{S} 0 \mathrm{D} 0 \mathrm{~B} 1 \mathrm{C} 0\right) .
\end{aligned}
$$

The expected cost is equal to $\$ 3.91$ and the costs incurred when $\boldsymbol{x}_{\mathrm{R}}$ is applied to the four likely scenarios are shown in 


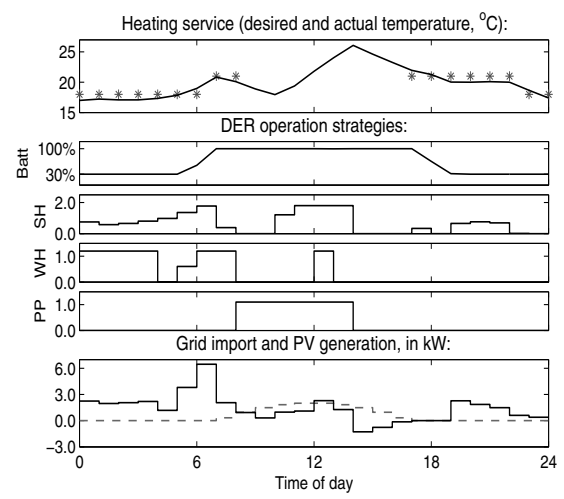

(a) $10 \%$ CPP probability

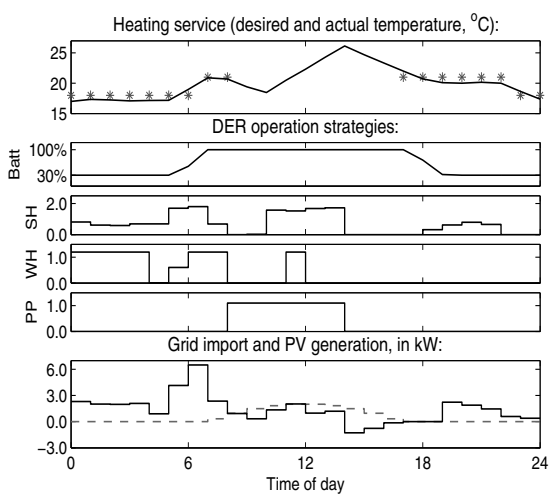

(b) $50 \%$ CPP probability

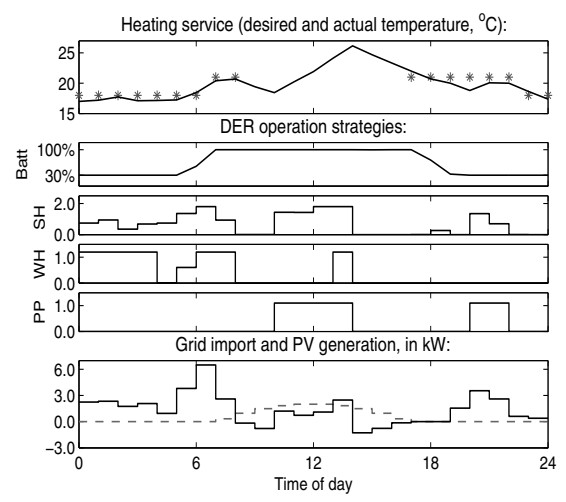

(c) $90 \%$ CPP probability

Fig. 4. Robust DER operation schedules for tariff ToU energy + ToU feed-in, sunny day, normal demand, and PHEV available as storage DER. Batt $=$ PHEV battery state of charge $(\%$ of capacity), $\mathrm{SH}=$ space heater power $(\mathrm{kW}), \mathrm{WH}=$ water heater power $(\mathrm{kW}), \mathrm{PP}=$ pool pump power $(\mathrm{kW})$.

TABLE V

COST INCURRED WHEN SCHEDULING UNDER SOLAR INSOLATION AND DEMAND ForeCAST UNCERTAINTY (\$)

\begin{tabular}{c|c|cccc||c}
\hline \multirow{2}{*}{$\begin{array}{c}\text { Actual } \\
\text { scenario }\end{array}$} & Assumed & \multicolumn{4}{|c||}{ Schedule for scenario } & Robust \\
& bility & S1D1 & S1D0 & S0D1 & S0D0 & schedule \\
& B1C0 & B1C0 & B1C0 & B1C0 & $\boldsymbol{x}_{\mathrm{R}}$ \\
\hline S1D1B1C0 & $48 \%$ & $\mathbf{4 . 3 2}$ & 8.22 & 4.80 & 8.53 & 4.37 \\
S1D0B1C0 & $32 \%$ & 2.75 & $\mathbf{2 . 1 1}$ & 3.20 & 2.49 & 2.18 \\
S0D1B1C0 & $12 \%$ & 6.41 & 10.23 & $\mathbf{6 . 0 2}$ & 9.75 & 6.46 \\
S0D0B1C0 & $8 \%$ & 4.77 & 4.15 & 4.31 & $\mathbf{3 . 6 3}$ & 4.24 \\
\hline
\end{tabular}

the last column of Table V. Columns 3 to 6 are constructed by implementing the schedules for the four possible scenarios to all four scenarios, similar to Table III. The actual costs are shown so they may be compared to the entries in the last column.

With respect to the end-users, the first two scenarios are most likely to occur, hence the costs on these scenarios if $\boldsymbol{x}_{\mathrm{R}}$ is used (\$4.37 and \$2.18) are close to the lowest costs possible (\$4.32 and \$2.11). The last one is the least likely to occur, so the resulting cost if this scenario occurs $(\$ 4.24)$ is quite far from the lowest cost possible (\$3.63). The robust schedule is also able to hedge the end-users against possible additional costs resulting from inaccurate predictions.

\section{CONCLUSION}

We were able to determine the value of accurate forecasts when formulating the DER operation schedules for a residential energy services provision case study. The values are determined by computing the avoided additional costs due to correct forecasts. In the 'smart' home case study, we were able to determine the value of making accurate solar insolation, energy services demand, PHEV availability and CPP status forecasts using two different tariff structures.

We also demonstrated how to use the scheduler to formulate robust DER schedules when the end-users cannot make accurate predictions. A robust schedule is formulated by maximizing the expected net benefit, and the perceived probability of occurrence of uncertain events are used to weigh the net benefits that result when the robust schedule is implemented on all possible scenarios. In the two examples, the robust schedules were able to hedge the end-users against large additional costs that may result when incorrect schedules are implemented.

\section{ACKNOWLEDGMENT}

Michael Angelo Pedrasa acknowledges the scholarship granted by the University of the Philippines and the Philippines Department of Science and Technology through the ERDT.

\section{REFERENCES}

[1] C.-H. Lien, Y.-W. Bai, and M.-B. Lin, "Remote-controllable power outlet system for home power management," IEEE Trans. Consum. Electron., vol. 53, no. 4, pp. 1634-1641, Nov. 2007.

[2] S. Rojchaya and M. Konghirun, "Development of energy management and warning system for resident: An energy saving solution," in 6th Int. Conf. on Elec. Eng./Electron., Comput., Telecommun. and Inform. Technology, vol. 1, May 2009, pp. 426-429.

[3] K. Balasubramanian and A. Cellatoglu, "Improvements in home automation strategies for designing apparatus for efficient smart home," IEEE Trans. Consum. Electron., vol. 54, no. 4, pp. 1681-1687, Nov. 2008.

[4] M. Pedrasa, E. Spooner, and I. MacGill, "Improved energy services provision through the intelligent control of distributed energy resources," presented at the 2009 IEEE Bucharest PowerTech, Jun/Jul 2009.

[5] S. Abras, S. Pesty, S. Ploix, and M. Jacomino, "An anticipation mechanism for power management in a smart home using multi-agent systems," presented at the 3rd Int. Conf. on Inform. and Commun. Technologies: From Theory to Applicat., 2008, Apr. 2008.

[6] R. Negenborn, M. Houwing, B. De Schutter, and H. Hellendoorn, "Adaptive prediction model accuracy in the control of residential energy resources," in 2008 IEEE Int. Conf. on Control Applicat., Sept. 2008, pp. 311-316.

[7] R. Hamalamen, J. Mantysaari, J. Ruusunen, and P.-O. Pineau, "Consumption strategies and tariff coordination for cooperative consumers in a deregulated electricity market," in Proc. of the 32nd Annual Hawaii Int. Conf. on Syst. Sciences, Jan. 1999.

[8] M. Pedrasa, T. Spooner, and I. MacGill, "Coordinated scheduling of residential distributed energy resources to optimize 'smart' home energy services," submitted for publication.

[9] J. Kennedy and R. Eberhart, "Particle swarm optimization," in Proc. 1995 IEEE Int. Conf. on Neural Networks, vol. 4, Nov/Dec 1995, pp. 1942-1948.

[10] F. van den Bergh and A. Engelbrecht, "A cooperative approach to particle swarm optimization," IEEE Trans. Evol. Comput., vol. 8, no. 3, pp. 225239, June 2004.

[11] J. Kennedy and R. Eberhart, "A discrete binary version of the particle swarm algorithm," in 1997 IEEE Int. Conf. on Syst., Man, and Cybern., vol. 5, Oct 1997, pp. 4104-4108. 